\title{
FÓRUM - REDES SOCIAIS E INTERORGANIZACIONAIS
}

\author{
Ana Cristina Braga Martes \\ FGV-EAESP \\ E-mail: acbmartes@gvsp.br
}

\author{
Sérgio Bulgacov \\ UFPR \\ E-mail: s.bulgaco@@ufpr.br
}

\author{
Mauricio Reinert do Nascimento \\ FGV- EAESP e Univali \\ E-mail:m.reinert@uol.com.br
}

\author{
Sandro Aparecido Gonçalves \\ FGV- EAESP \\ E-mail: sandro.nadia@uol.com.br

\section{Paulo Mussi Augusto} \\ FGV- EAESP e PUC-PR \\ E-mail: paulomaugusto@uol.com.br
}

Entre os estudos pioneiros sobre redes sociais, destacam-se dois trabalhos: The Strength of Weak Ties, publicado em 1973, e posteriormente G etting a Job, publicado em 1974, ambos do sociólogo norte-americano M ark Granovetter. N eles, o autor identifica formas de acesso aos postos de trabal ho e conclui que são os "conhecidos" (como, por exemplo, ex-colegas de graduação) e não amigos próximos ou familiares (os quais conformam laços sociais mais fortes) que, de fato, promovem 0 acesso de pessoas aos postos de trabal ho disponíveis. Isso porque aqueles que pertencem a um mesmo círculo social (mais íntimo) compartilham entre si o mesmo tipo e número de informações sobre oportunidades de emprego e contatos. No primeiro caso (laços sociais fortes), as informações disponibilizadas são redundantes, enquanto no segundo (laços fracos), abrem-se possibilidades de informações novas e mais abrangentes. Com esses trabalhos, Granovetter introduziu a discussão sobre a importância e o papel das redes na vasta bibliografia sobre mercado de trabalho, assim como o papel das redes na promoção e acesso a oportunidades existentes na estrutura social e econômica.
Pesquisas como as de Granovetter deram enorme impulso aos estudos sobre redes sociais nos últimos anos. Contudo, tal crescimento decorre de uma mudança maior no mundo acadêmico, com início na segunda metade do século XX: a fuga de explicações individualistas, essencial istas e atomistas em direção a explicações relacionais, contextuais e sistêmicas. Trata-se de uma mudança que vem ocorren do em diversos campos do saber (como nos Estudos Organizacionais, Sociologia, Antropologia, até a Medicina e a Física) e cujas origens remontam aos meados do século $X X$.

De fato, ao se analisar a evolução histórica da análise de redes, especialmente dentro da tradição sociológica, podem-se identificar três bases formadoras: estudos sociométricos, apoiados na psicol ogia gestáltica; estudos desenvolvidos por antropólogos da Universidade de Manchester; e estudos desenvolvidos pelos estruturalistas da Universidade Harvard. A análise de redes que utiliza estudos sociométricos tem seu marco nos trabal hos de Jacob M oreno, desenvolvidos durante a década de 1930. M oreno utilizava sociogramas - representando grupos como uma coleção de pontos 
conectados por linhas - para identificar os relacionamentos em forma de rede entre pessoas e os padrões de interação, clusters e a dinâmica de pequenos grupos. 0 grupo de Manchester, por sua vez, era integrado por uma grande variedade de pesquisadores daquela universidade, que alcançaram avanços consideráveis ao aliar a matemática com a teoria social substantiva, no final da década de 1950 (Scott, 2003). Duas abordagens podem ser creditadas a este grupo: a utilização das redes egocêntricas, isto é, a análise de redes em torno de um indivíduo em particular, e a análise do conteúdo dos laços da rede por meio de abstrações que descrevem modos particulares de atividade social, tais como parentesco, interação política, amizade e relações de trabal ho. Já os estudos desenvolvidos na U niversidade $\mathrm{H}$ arvard por Harrison W hite e seus estudantes no final dos anos 1960 conciliaram diferentes tradições da análise de redes nas suas investigações sobre a base matemática das estruturas sociais (Scott, 2003). Sua proposta de modelar e mensurar matematicamente os papéis sociais - chamada de blockmodeling pode ser considerada como o fundamento da atual forma de análise de redes.

A relação entre redes e empresas começa a ser estudada no final dos anos 1970, quando economistas e sociólogos passaram a investigar, entre outros aspectos, os fluxos de informação como sendo processos de formação de redes [networking process], para compreender seu significado para o âmbito corporativo. Destacam-se os trabalhos de Paul DiMaggio e Walter Powell, que enfocam as relações entre redes e informação no campo da sociologia das organizações, especialmente o processo de difusão de práticas organizacionais em ramos específicos. Para eles, o comportamento de uma empresa é também modelado pelo comportamento de outras organizações consideradas exemplares.

Firmas não estão apenas inseridas em uma intrincada rede de relações com outras organizações, mas também respondem a ações de firmas de alta visibilidade e prestígio no seu campo respectivo. As organizações pioneiras na adoção de práticas inovadoras mais provavelmente estarão situadas na intersecção de múltiplas redes, ligadas a fontes informacionais diversas, que as expõe mais rapidamente em contato tanto com novas idéias emergentes, quanto a eventuais avaliações críticas sobre seus méritos. Tomadas em conjunto, as informações disponíveis através das redes profissionais e de status, modelam as definições sobre os tipos de comportamento que são apropriados e sobre os padrões que as organizações procuraram al cançar. (POWELL e SMITH-DOERR, 1994, p. 376).

Especificamente no âmbito da teoria das organizações, a perspectiva de redes sociais emergiu no final dos anos 1970, quando autores como Aldrich (1979) e Williamson (1975) passaram a tomar as formas de relacionamento interorganizacional como foco de análise. Tichy, Tushman e Fombrun (1979) utilizaram-se da anál ise de rede no âmbito dos estudos organizacionais com o objetivo de compreender o comportamento das organizações. Isso porque consideram tal abordagem capaz de englobar as mais diversas interações (tanto individuais como organizacionais) e possibilitar a identificação das causas e conseqüências da estrutura dos relacionamentos, assim como sua padronização. Também na área de sociol ogia econômica pesquisadores passam a utilizar a abordagem de redes como uma forma alternativa à abordagem "mercados e a hierarquia" para explicar as regras que orientam as diversas transações interorganizacionais (Jarillo, 1988; Powell, 1990; Thorelli, 1986).

A perspectiva analítica do estudo de redes reforçou e expandiu a sociologia econômica e, ao mesmo tempo, renovou um conjunto de questionamentos sobre importantes pressupostos da teoria econômica ortodo$x$ a. Tomar as redes sociais como foco pressupõe que as próprias organizações e transações econômicas estejam imersas [embedded] em uma rede de relações sociais. Diferentes tipos de imersão, ou envolvimento com demais redes, podem facilitar ou gerar constrangimentos à ação organizacional (ou do agente econômico) e, portanto, orientam a formação de interesses e a tomada de decisões. De acordo com Granovetter, os atores se comportam e tomam decisões dentro de um contexto social determinado e não devem ser tomados como átomos isolados, da mesma forma que não aderem a um roteiro pré-escrito. Ao contrário, as organizações agem no interior de um sistema de relações concretas e em permanente desenvolvimento (Granovetter, 1985). Como decorrência, torna-se crucial abarcar 0 ambiente na análise das organizações e elaborar estudos predicativos, mas de um modo al ternativo àquele dos estudos econômicos tradicionais: "Enquanto os estudos econômicos têm analisado tais comportamentos como respostas competitivas em um contexto de oligopólios, a teoria das redes tem enfatizado o papel do comportamento imitativo impelido pela incerteza ou status de conformidade" (Nohria e 
Gulati, 1994, p. 454). Em resumo, a abordagem de redes representa uma alternativa tanto ao determinismo cultural, na medida em que as relações entre indivíduos e não indivíduos isolados é que são tomadas como unidade de análise (Powell e Smith-Doerr, 1994), como ao individualismo atomizado da abordagem econômica neoclássica, porque as redes são entendidas como produto da human agency.

Estudos de (e sobre) redes delimitam tipos al ternativos de abordagem, ancorados em pelo menos dois diferentes conceitos de rede. De um modo geral, o conceito de rede tende a ser descritivo: uma rede é composta por um conjunto de relações ou laços entre atores (indivíduos ou organizações). Granovetter define redes sociais como um "conjunto de nós ou atores ( pessoas ou organizações) ligados por relações sociais ou laços de tipos específicos. U m laço ou relação entre dois autores tem força [strength] e conteúdo. 0 conteúdo inclui informação, consel ho ou amizade, interesses compartilhados ou pertencimentos e, tipicamente, algum nível de confiança" (Granovetter et al., 1998, p. 219).

Mas as redes também podem ser concebidas como forma de governança, tal como preconizam Michael Piore e Charles Sabel (1984). A partir dos anos 1970, diante do problema de saturação do mercado de produtos de massa, as empresas começam a flexibilizar sua produção e passam a trabal har em redes. Rapidamente a produção flexível se mostra dotada de capacidades adicionais: alterar rapidamente seus produtos em função das novas tecnologias de automação, e integrar outros fornecedores e distribuidores por meio de processos de tercei rização e novos tipos de contratação. Essa nova lógica de produção, num ambiente versátil e competitivo, é al tamente dependente do trabalho em rede entre as firmas que compõem a cadeia de produção e distribuição (Piore e Sabel, 1984).

$\mathrm{N}$ a literatura internacional, especialmente na angl osaxã, podem ser encontrados pelo menos quatro princípios inter-relacionados que têm sido utilizados para gerar teorias e hipóteses de pesquisa: (1) a importância das relações entre atores; (2) a imersão social dos atores [embeddness] em campos sociais; (3) a utilidade das conexões da rede (capital social); e (4) a padronização estrutural da vida social (Kilduff e Tsai, 2003). A ênfase nas relações entre atores é o fator distintivo mais marcante da perspectiva de redes dentro dos estudos organizacionais. De fato, a principal característica das análises de redes mais recentes recai sobre a importância das interações en tre os atores, em lugar de focalizar seus atributos, como caráter explicativo das ações individuais. Essa proposta pressupõe que os relacionamentos são constitutivos da natureza humana e que el es são el ementos definidores da identidade dos atores sociais. A imersão social das ações econômicas pode ser vista como um segundo princípio distintivo da pesquisa de redes dentro dos estudos organizacionais. Ao se utilizar esse princípio, assumese que o comportamento humano está imerso dentro de uma rede de relacionamentos interpessoais, e isso se estende às transações econômicas e ao mundo corporativo (Granovetter, 1985; Uzzi, 1996). O terceiro princípio refere-se à crença de que as conexões dentro de uma rede constituem uma espécie de capital social que pode prover valor, inclusive econômico, aos integrantes da rede (Burt, 1992). O quarto princípio está relacionado aos fatores estruturais que devem ser analisados para identificar quais atores geram e criam laços em uma determinada rede. Redes de organizações podem ser analisadas pelo grau de agrupamento [clustering] e pelo grau de distanciamento dos atores que a ela pertencem.

Em estudo realizado por Martes et al. (2005) - que faz uma análise da literatura internacional sobre o tema "redes sociais e empresas" compreenden do o período de 1990 a 2002 - foram encontrados 14 periódicos das áreas de Administração, Sociologia e Economia que tratavam do tema em al guma de suas edições. A lista de periódicos é a seguinte: Academy of Management Journal, Academy of Management Review, Administrative Science Quarterly, American Journal of Sociology, American Sociological Review, Annual Review of Sociology, Economic G eography, Journal of International Business Studies, Journal of Social Structure, Management Science, Organization Science, Organization Studies, Rand Journal of Economics e Strategic M anagement Journal. A pesquisa identificou 224 artigos que, além de trabal harem com redes, tinham como tema as relações estabelecidas entre as empresas e os locais onde estão inseridas; relações entre redes de empresa e mudança organizacional; e a relação das redes de empresas na definição da estratégia organizacional. Os artigos totalizaram uma média de quase 19 artigos por ano, com grande concentração nos anos 1996 e 1998, com o lançamento de edições especiais em diversos periódicos, evidenciando sua importância crescente.

Dentre os artigos identificados, 82 referem-se a redes e inserção local. De um modo geral, el es destacam a importância das relações locais nas decisões de migração de empresas e na constituição de algum tipo de 
legitimidade social. Um dos aspectos mais inovadores desses estudos refere-se à interligação entre os dirigentes das organizações, mais especificamente à participação de diretores de uma organização no conseIho de administração de outras organizações (Scott, 1991; Mizruchi, 1996). Esse tipo de interligação proporciona acesso à informação e poder (Scott, 1991), além de servir como instrumento de controle, ou como estrutura de governança ( Maman, 1999). A existência de interligação de diretores com organizações locais possibilita maior troca de informação e fortalecimento dos laços, e pode indicar maior inserção local. Mizruchi (1996) acrescenta que a interligação de diretores pode ser utilizada como indicador de imersão social, o que equivale a dizer que a análise de redes pode ser ampliada para as relações das empresas com outras organizações, tais como universidades e associações profissionais. São essas possibilidades que transformam a teoria de redes em uma oportunidade para a Sociologia E conômica e para a Teoria das O rganizações aprofundarem seus conhecimentos sobre participação das empresas na construção do ambiente em que estão inseridas.

Sobre o tema "estratégia organizacional e redes" foram encontrados 70 artigos. Eles convergem para a explicação: a competição é mais bem explicada pelo posicionamento de uma empresa dentro da rede do que como um "ataque" ao ambiente. A rede é entendida, então, como um arranjo que pode ser manejado intencionalmente com o objetivo de obter vantagem competitiva. 0 princípio orientador do desenvolvimento de redes estratégicas passa a ser o entendimento de como e quando o comportamento cooperativo deve superar o comportamento competitivo. Sobre o tema sobre mudança e inovação foram encontrados 72 artigos. De maneira geral, os artigos buscam esclarecer a dinâmica de formação dos vínculos sociais. M udanças organizacionais, incluindo-se desde a adaptação a exigências ambientais até a inovação tecnológica, explorando-se as redes interorganizacionais por meio de proposições normativas, caracterizam esses artigos.

Em síntese, a produção internacional sobre redes e organizações apresenta um rápido crescimento nos últimos anos, incorporando elementos de diferentes tradições para gerar novas teorias e hipóteses orientadoras de pesquisas empíricas. Devido à sua importância, ao seu potencial e ao seu volume de produção podese dizer que esse tema já compõe o mainstream da área.

Publicações sobre redes na área de Administração tomam impulso na década de 1970 e 1980 nos Esta- dos Unidos e Inglaterra, mas não chegam ao Brasil antes dos anos 1990. Todavia, o interesse pelo tema vem crescendo, acompanhando o crescimento das pesquisas acadêmicas em Administração.

Em pesquisa real izada em sete periódicos nacionais ${ }^{1}$ ( $M$ artes et al. 2005) sobre o tema, foram encontrados 18 artigos entre os anos de 1990 e 1996 (em média três artigos por ano) e 51 artigos entre os anos de 1997 e 2006 (média de 5,1 por ano). Os números tornam evidente a crescente importância do tema nas universidades brasileiras. O bserva-se, ainda, uma concentração da produção nacional, pois a RAE-revista de administração de empresas, a RAC - Revista de Administração Contemporânea e a RAP - Revista de Administração Pública juntas representaram $65,3 \%$ da produção a partir da segunda metade dos anos 1990.

É possível classificar os artigos nacionais dentro da "clássica" divisão da área: (1) análise estrutural de redes sociais e (2) abordagem de redes (estrutura de governança). No primeiro grupo, a pesquisa encontrou artigos que focalizam as relações sociais, capital social, laços fortes e fracos que se estabelecem entre os atores, como também aquel es que trabal ham com a construção topográfica da rede, empregando metodologia específica para medir intensidade, grau de conectividade, dinâmica e disposição espacial dos casos analisados. No segundo grupo são encontradas pesquisas que, distanciando-se em certa medida do conceito de redes sociais, estão preocupadas com estruturas corporativas que assumem a forma de redes, abordando as redes interorganizacionais como estruturas de governança.

Faz-se necessário destacar que na área de Administração ainda são poucos os trabalhos nacionais que fazem uso da metodologia específica de rede para analisar intensidade e características estrutu rais das relações, o que difere sobremaneira da produção anglosaxônica, em que é bastante forte esse tipo de pesquisa. No que se refere aos temas abordados, merecem destaque elaboração e fluxo de conhecimento, cooperação, confiança, desenvolvimento regional a partir de redes de pequenas e médias empresas, competitividade a partir das inter-relações de grandes corporações, em especial as alianças estratégicas, benefícios advindos da troca de informação, internacionalização de empresas, governança corporativa, mecanismos de controle do setor público e formas de participação da sociedade civil, entre outras coisas.

Para compor esta edição, a RAE selecionou quatro artigos, além do artigo internacional convidado. Os 
textos demonstram haver singularidades relevantes no interior desse grande tema, mas também diversidades nas suas composições tanto em termos de área de abrangência como nos procedimentos da investigação, ou ainda na forma de apresentação dos resultados. 0 primeiro artigo, de Mariana Baldi e Marcelo Milano Falcão Vieira, faz um estudo de caso longitudinal intitulado "Calçado do Vale: imersão social e redes interorganizacionais", que discute de que modo os tipos de laços, a oposição e a arquitetura da rede afetam a ação econômica do setor coureiro-calçadista do Vale do Rio dos Sinos do Rio Grande do Sul. O segundo artigo, de Sérgio Luís Boeira, intitulado "Indústria de tabaco versus cidadania: confronto entre redes organizacionais", utiliza a perspectiva histórica da indústria de tabaco e da formação de sua rede estratégica e social, analisando as condições de crescimento e contradições existentes na rede estratégica empresarial e na rede antifumo. 0 terceiro estudo, de Herbert Kimura, Maria Luisa Mendes Teixeira e Arilda Schmidt Godoy, intitulado "Redes sociais, valores e competências: simulação de conexões", evidencia, por meio de simulações, os efeitos da competência e do consenso sobre a dinâmica das interações e da formação de conexões entre indivíduos, como fatores de aproximação e de facilitação de conexões nas redes sociais. 0 quarto artigo, de Charles Kirschbaum, "Renascença da indústria brasileira de filmes: destinos entrelaçados?", utiliza-se da perspectiva estratégica, sob o foco da vantagem competitiva, para reconstruir a rede da indústria brasileira de filmes de 1994 a 2002. 0 texto analisa os preditores do desempenho individual e das variáveis estratégicas, em diferentes períodos, sob a perspectiva em rede da indústria do cinema ainda em desenvolvimento.

Além desses quatro artigos nacionais, segue-se também 0 artigo de Mark Mizruchi intitulado "Análise de redes sociais: conquistas recentes e controvérsias atuais". Mizruchi é professor do Departamento de Sociologia da Escola de Administração da Universidade de Michigan (Ann Arbor/Estados Unidos). Atualmente seus interesses de pesquisa estão direcionados para a compreensão do comportamento econômico e político das grandes corporações norte-americanas, utilizando a análise de redes sociais. Com três livros publicados sobre o tema e mais de 80 artigos acadêmicos, Mizruchi é hoje referência obrigatória nas pesquisas sobre redes e sociologia econômica nos Estados Unidos. Seu artigo apresenta um breve histórico sobre análise de redes, uma síntese sobre os métodos utili- zados e suas aplicações em pesquisa sobre poder e relacionamentos interorganizacionais. 0 mais interessante, todavia, são as controvérsias sobre as diferentes perspectivas de redes discutidas na segunda parte do artigo. A primeira controvérsia diz respeito à relação entre análise de redes e teoria da escolha racional. A segunda refere-se ao papel da cultura e dos aspectos normativos na teoria de redes. A terceira se volta para as dificuldades de se inserir a ação humano [human agency] nas análises. A pesar desses desafios, o autor destaca que a análise de redes tem crescido justamente por conseguir reter uma concepção mais dinâmica das ações sociais e possibilitar que se trabalhe em conjunto com outras teorias.

Acreditamos que os cinco artigos, que agora se seguem, poderão contribuir para o fortalecimento da abordagem de redes no Brasil, seja no campo da Teoria Organizacional, da Teoria Institucional ou ainda da Sociologia Econômica.

\section{NOTA}

\footnotetext{
${ }^{1}$ Revista de Administração de Empresas (RAE), Revista de Administração Contemporânea (RAC), Revista de Administração da USP (RAUSP), Revista de Administração Pública (RAP), Revista de Economia Política (REP), Organizações \& Sociedade (O\& S) e Revista Brasileira de Ciências Sociais.
}

\section{REFERÊNCIAS BIBLIOGRÁFICAS}

BURT, R. S. Structural Hole. Cambridge, MA: Harvard University Press, 1992.

GARCIA-PONT, C.; NOHRIA, N. Local versus global mimetism: the dynamics of alliance formation in the automobile industry. Strategic $\mathrm{Ma}$ nagement Journal, v. 23, n. 4, p. 307-321, 2000.

GRAN OVETTER, M. Economic action and social structure: the problem of embeddedness. American Journal of Sociology, v. 91, n. 3, p. 481-510, 1985.

GRAN OVETTER, M. Getting a Job. Cambridge: Harvard University Press, 1974.

GRANOVETTER, M. The Strength of Weak Ties. American Journal of Sociology, v. 78, n. 6, p. 1360-80, 1973.

GRANOVETTER, M.; CASTILLA, E.; HWANG, H. GRANOVETTER, E. Social networks in Silicon Valley. In: LEE, C. M.; MILLER, W. F.; 
HANCOCK, M. G.; ROWEN, H. S. (Eds.). TheSilicon Valley Edge. Stanford: Stanford University Press, 2000. p. 218-247.

GRANOVETTER, M.; SWEDBERG, R. The Sociology of Economic Life. Cambridge: Westview, 2001.

GULATI, R. Alliances and networks. Strategic Management Journal, v. 19, n. 4, p. 293-317, 1998.

GULATI, R. Social structure and alliance formation patterns: a longitudinal analysis. Administrative Science Quarterly, v. 40, n. 4, p. 619-652, 1995.

GULATI, R.; NOHRIA, N.; ZAHAEER, A. Strategic networks. Strategic Management Journal, v. 21, n. 3, p. 203-215, 2000.

JARILLO, J. On strategic networks. Strategic M anagement Journal, v. 9, n. 1, p. 31-41, 1988.

NOHRIA, N.; ECCLES, R. G. (Orgs.). N etwork and organizations: structure, form and action. Boston: Harvard Business School Press, 1992.

MARTES, A. C. B. et al. Redes sociais e empresas. Relatório de pesquisa. Núcleo de Pesquisa e Publicações, EAESP/N PP/FGV, 2005.

MIZRUCHI, M. What do interlocks do? An analysis, critique, and assessment of research on interlocking directorates. Annual Review of Sociology, v. 22, p. 271-298, 1996.
POWELL, W.; KPOUT, K.; SMITH-DOERR, L. Interorganizational collaboration and the locus of innovation: networks of learning in biotechnology. Administrative Science Quarterly, v. 41, n. 1, p. 116-145, 1996.

SABEL, C.; PIORE, M. The Second Industrial Divide. N ew York: Basic Books, 1984.

SCOTT, J. N etworks of corporate power: a comparative assessment. Annual Review of Sociology, v. 17, p. 181-203, 1991.

SMITH, K.; CARROL, S.; ASHFORD, S. Intra- and interorganizational cooperation: toward a research agenda. A cademy of M anagement Journal, $v$. 38, n. 1, p. 7-23, 1995.

TICHY, N; TUSHMAN, M; FOMBRUM, C. Social network analysis for organizations. Academy of M anagement Review, v. 4, n. 4, p. 507-519, 1979.

UZZI, B. Social structure and the interfirm networks: the paradox of embeddedness. Administrative Science Quaterly, v. 42, n. 1, p. 35-67, 1997.

UZZI, B. The sources and consequences of embededdness for the economic performance of organizations: the network effect. American Sociological Review, v. 61, p. 674-698, 1996.

\section{Artigo convidado. Aprovado em 01.06.2006}

